\title{
Interpretations of Support Among Arguments
}

\author{
Liuwen YU ${ }^{\mathrm{a}, \mathrm{b}, \mathrm{c}, 1}$, Réka MARKOVICH ${ }^{\mathrm{a}}$ and Leendert VAN DER TORRE ${ }^{\mathrm{a}, \mathrm{d}}$ \\ ${ }^{a}$ University of Luxembourg, Luxembourg \\ ${ }^{\mathrm{b}}$ University of Bologna, Italy \\ ${ }^{\mathrm{c}}$ University of Turin, Italy \\ ${ }^{\mathrm{d}}$ Zhejiang University, China
}

\begin{abstract}
The theory of formal argumentation distinguishes and unifies various notions of attack, support and preference among arguments, and principles are used to classify the semantics of various kinds of argumentation frameworks. In this paper, we consider the case in which we know that an argument is supporting another one, but we do not know yet which kind of support it is. Most common in the literature is to classify support as deductive, necessary, or evidentiary. Alternatively, support is characterized using principles. We discuss the interpretation of support using a legal divorce action. Technical results and proofs can be found in an accompanying technical report.
\end{abstract}

Keywords. formal argumentation, abstract argumentation, bipolar argumentation, principle-based approach, legal interpretation

\section{Introduction}

The theory of formal argumentation distinguishes and unifies different notions of attack, support and preference among arguments. For example, whereas in structured argumentation attack among arguments can be based on rebut, undercutting or undermining, at the abstract level all these kinds of attack are treated in a uniform way. Likewise, deductive, necessary and evidentiary support can be unified at the abstract level [14]. The picture that emerges from the formal argumentation literature is that there is a broad agreement on how to interpret attack, even when different kinds of semantics have been proposed, whereas there is less consensus on the interpretation of support. Moreover, different kinds of support can occur in the same framework, and each variant of support can be more prominent in a particular application.

The common approach in the literature classifies a support among arguments as deductive support, necessary support or evidentiary support. Deductive support [2] captures the intuition that if $a$ supports $b$, then the acceptance of $a$ implies the acceptance of $b$, and as a consequence the non-acceptance of $b$ implies the non-acceptance of $a$. Evidentiary support $[11,10]$ distinguishes prima-facie from standard arguments, where prima-facie arguments do not require any support from other arguments to stand, while standard ar-

\footnotetext{
${ }^{1}$ This project has received funding from the European Union's Horizon 2020 research and innovation program under the Marie Skłodowska-Curie ITN EJD grant agreement No 814177.
} 
guments must be supported by at least one prima-facie argument. Necessary support [9] captures the intuition that if $a$ supports $b$, then the acceptance of $a$ is necessary to get the acceptance of $b$, or equivalently the acceptance of $b$ implies the acceptance of $a$.

In addition to this classification, we can consider the principles the support relation satisfies. In formal argumentation, principles can be used as a guideline for choosing the appropriate definitions and semantics depending on various needs. After the principles are chosen, it can be seen at a second step whether there is a semantics satisfying that set of principles. If a set of principles corresponds to one of the semantics then the support can be classified as such, but it may also be the case that no semantics corresponds to the desired set of principles.

In this paper, we consider the case in which an argument supports another one, but we do not know yet which kind of support it is. We consider a legal divorce action in which the interpretation of support is in close relation to the interpretation of law itself. In a divorce action, a judge should decide about the custody according to the child's best interest. The civil code says that, when deciding, the judge has to take the child's opinion into consideration. We also show how the interpretation of this rule influences the interpretation of the support relation, and how this latter interpretation influences the judgement.

Our paper contributes to the discussion on the formalization of legal interpretation in the following way. The role of interpretation is crucial in law, but it is also a source of criticism of using logic-based methods in modelling legal reasoning. For example, Prakken reminds to Leith's warning that the knowledge-engineer's interpretation when formalizing is necessarily premature, as the authority of interpretation of law is assigned to the judiciary [16]. Addressing this criticism, the literature on legal interpretation has discussed the possibility that legal knowledge-based systems contain alternative syntactic formalizations. Prakken observes that while on the syntactic level formalization commits us to a given interpretation, on the conceptual level, classification of factual situations as legal concepts is not an issue of logical form [16, p.14]. Alternatively, we can restrict the investigation by saying that "the only aspects of legal reasoning which can be formalized are those aspects which concern the following problem: given a particular interpretation of a body of legal knowledge, and given a particular description of some legal problem, what are then the general rational patterns of reasoning with which a solution to the problem can be obtained?" [16, p.4]. If a formal framework itself offers the different interpretations, though, then using it might be directly exploitable to the comparison of the different possibilities and routes of reasoning given each interpretation.

In a recent paper, Prakken [18] argues that for the validation of bipolar argumentation theory, the so-called theory-based validation is preferred to an empirical validation [13], which itself is preferred to an intuition-based validation. We agree with this ordering, but we believe that the principle-based analysis complements these validation methods, and that the theory of formal argumentation needs to be complemented with examples and case studies about the use of the theory. This paper contributes to the latter two areas.

The layout of this paper is as follows. In Section 2 we briefly repeat the definitions of bipolar argumentation, and we introduce a principle-based approach. In Section 3 we apply the theory to the legal divorce action. All technical details and proofs of this paper can be found in a technical report [20], and will be added to the journal extension of this paper. 


\section{Formal approaches to the interpretation of support among arguments}

For completeness we repeat the basic definitions of abstract argumentation theory.

Definition 1 (Dung semantics [6]) An argumentation framework $(A F)$ is a tuple $\langle\mathcal{A}, \mathcal{R}\rangle$ where $\mathcal{A}$ is a set of arguments and $\mathcal{R} \subseteq \mathcal{A} \times \mathcal{A}$ is a binary attack relation over $\mathcal{A}$. $E \subseteq \mathcal{A}$ is conflict-free iff $\nexists a, b \in E$ such that $(a, b) \in \mathcal{R} . E \subseteq \mathcal{A}$ defends $c$ iff $\forall b \in \mathcal{A}$ with $(b, c) \in \mathcal{R}, \exists a \in E$ such that $(a, b) \in \mathcal{R} . E \subseteq \mathcal{A}$ is admissible iff it is conflictfree and defends all its elements. A conflict-free $E \subseteq \mathcal{A}$ is a complete extension iff $E=\{a \mid E$ defends $a\} . E \subseteq \mathcal{A}$ is the grounded extension iff it is the smallest (for set inclusion) complete extension. $E \subseteq \mathcal{A}$ is a preferred extension iff it is the largest (for set inclusion) complete extension. $E \subseteq \mathcal{A}$ is a stable extension iff it is admissible and attacks all arguments in $\mathcal{A} \backslash E$.

The semantics of deductive and necessary support is based on a reduction of a bipolar framework to an argumentation framework together with a Dung semantics. Based on various interpretations, these reductions add indirect attacks obtained from sets of attack and support relations, then from the obtained indirect attacks and the support additional indirect attacks can be added and so on. We follow the style and notation of Polberg [12], and the reductions are visualized in Figure 1.

Definition 2 (Deductive and necessary support [3,12]) $A$ bipolar argumentation framework (BAF, for short) is a 3-tuple $\langle\mathcal{A}, \mathcal{R}, \mathcal{S}\rangle$, adding a binary support relation $\mathcal{S} \subseteq \mathcal{A} \times \mathcal{A}$ to $A F$ s. In addition six reductions from $B A F$ to $A F$ are defined:

SupportedReduction: $R S(B A F)=\left(\mathcal{A}, \mathcal{R} \cup \mathcal{R}^{\text {sup }}\right), \mathcal{R}^{\text {sup }}=\{(a, b) \mid(a, c)$ is in the transitive closure of $\mathcal{S},(c, b) \in \mathcal{R}\}$;

MediatedReduction: $R M(B A F)=\left(\mathcal{A}, \mathcal{R} \cup \mathcal{R}^{\text {med }}\right), \mathcal{R}^{\text {med }}=\{(a, b) \mid(b, c)$ is in the transitive closure of $\mathcal{S},(a, c) \in \mathcal{R}\}$;

SecondaryReduction: $R 2(B A F)=\left(\mathcal{A}, \mathcal{R} \cup \mathcal{R}^{s e c}\right), \mathcal{R}^{s e c}=\{(a, b) \mid(c, b)$ is in the transitive closure of $\mathcal{S},(a, c) \in \mathcal{R}\}$;

ExtendedReduction: $R E(B A F)=\left(\mathcal{A}, \mathcal{R} \cup \mathcal{R}^{\text {exd }}\right), \mathcal{R}^{\text {exd }}=\{(a, b) \mid(c, a)$ is in the transitive closure of $\mathcal{S},(c, b) \in \mathcal{R}\}$;

DeductiveReduction: $R D(B A F)=\left(\mathcal{A}, \mathcal{R} \cup \mathcal{R}^{\text {sup }} \cup \mathcal{R}_{\mathcal{R}^{\text {sup }}}^{\text {med }}\right)$, where $\mathcal{R}_{\mathcal{R}^{\text {sup }}}^{\text {med }}=\{(a, b) \mid(b, c)$ is in the transitive closure of $\mathcal{S},(a, c) \in \mathcal{R}$ or $\left.(a, c) \in \mathcal{R}^{\text {sup }}\right\}$;

NecessaryReduction: $R N(B A F)=\left(\mathcal{A}, \mathcal{R} \cup \mathcal{R}^{\sec } \cup \mathcal{R}^{\text {ext }}\right)$.

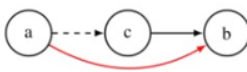

(a) Supported attack



(b) Mediated attack

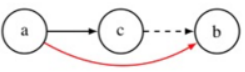

(c) Secondary attack

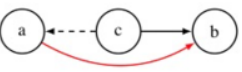

(d) Extended attack

Figure 1. Four kinds of indirect attacks as an intermediate step towards semantics for BAFs

We write $\mathcal{E}_{S R}$ for the function from BAF to sets of extensions, $\mathcal{E}_{S R}(B A F)=$ $\mathcal{E}_{S}(R(B A F)$ ), where $S$ is one of the Dung semantics (grounded, complete, preferred or stable) and $\mathrm{R}$ is one of the reductions (RS, RM, R2, RE, RD or RN). Thus we have $6 \times 4$ BAF semantics.

We represent evidentiary support using self-supporting arguments, see the technical report [20] for the comparison with other kinds of formalizations of evidentiary support. 
The basic idea is that every argument in the extension and every attacker is now an evidentiary chain from a self-supporting argument, a is self-supporting represented as $(a, a) \in \mathcal{S}$.

Definition 3 (Evidentiary support) Given a $B A F=\langle\mathcal{A}, \mathcal{R}, \mathcal{S}\rangle$. A sequence $\left(a_{0}, \ldots, a_{n}\right)$ of elements of $\mathcal{A}$ is an evidentiary sequence for argument $a_{n}$ iff $\left(a_{0}, a_{0}\right) \in \mathcal{S}$, and for $0 \leq i<n$ we have $\left(a_{i}, a_{i+1}\right) \in \mathcal{S}$. A set of arguments $S \subseteq \mathcal{A}$ e-defends argument $a \in \mathcal{A}$ iff for every evidentiary sequence $\left(a_{0}, \ldots, a_{n}\right)$ where $a_{n}$ attacks $a$, there is an argument $b \in S$ attacking one of the arguments of the sequence. Moreover, a set of arguments $S$ is e-admissible iff for every argument $a \in S$ there is an evidentiary sequence $\left(a_{0}, \ldots, a\right)$ such that each $a_{i} \in S$ ( $a$ is e-supported by $S$ ), $S$ is conflict free, and $S$ e-defends all its elements. A set of arguments is an e-complete extension iff it is e-admissible and it contains all arguments it e-defends; it is e-grounded extension iff it is a minimal e-complete extension; and it is e-preferred if it is maximal e-admissible extension. Moreover, it is $e$-stable if for every for every evidentiary sequence $\left(a_{0}, \ldots, a_{n}\right)$ where $a_{n}$ not in $S$, we have an argument $b \in S$ attacking an element of the sequence.

We introduce various principles for bipolar argumentation. Transitivity (P1, TRA) and closure (P2, CLO) of an extension under supported arguments are introduced by Cayrol et al. [4], inverse closure (P3) was introduced by Polberg [12]. Number of extensions (P4) says that adding support relations can only lead to a decrease of extensions. BAF directionality (P5) is a generalization of Dung's central principle of directionality. Global support (P6) and grounded support (P7) are two ways to characterize evidentiary support. Some additional principles are defined in the technical report [20].

Definition 4 (Principles) Given a $B A F=\langle\mathcal{A}, \mathcal{R}, \mathcal{S}\rangle$, a set $U$ is unattacked and unsupported if and only if there exists no $a \in \mathcal{A} \backslash U$ such that a attacks an argument of $U$ or a supports an argument of $U$. The set of all sets unattacked and unsupported arguments in $B A F$ is denoted $U S(B A F)$. A semantics $\mathcal{E}_{S R}$ satisfies principle $P$ iff for all $B A F$, for all $E$ in $\mathcal{E}_{S R}(B A F)$, we have:

P1. Transitivity If $(a, b) \in \mathcal{S},(b, c) \in \mathcal{S}$, then $\mathcal{E}_{S R}\langle\mathcal{A}, \mathcal{R}, \mathcal{S}\rangle=\mathcal{E}_{S R}\langle\mathcal{A}, \mathcal{R}, \mathcal{S} \cup(a, c)\rangle$.

P2. Closure If $(a, b) \in \mathcal{S}$ and $a \in E$, then $b \in E$.

P3. Inverse Closure If $(a, b) \in \mathcal{S}$ and $b \in E$, then $a \in E$.

P4. Number of extensions For all $\mathcal{S}^{\prime},\left|\mathcal{E}_{S R}\left(\mathcal{A}, \mathcal{R}, \mathcal{S} \cup \mathcal{S}^{\prime}\right)\right| \leqslant\left|\mathcal{E}_{S R}(\mathcal{A}, \mathcal{R}, \mathcal{S})\right|$.

P5. BAF Directionality $U \in U S(B A F)$, it holds that $\sigma\left(B A F_{\downarrow U}\right)=\{E \cap U \mid E \in \sigma(B A F)\}$, $B A F_{\downarrow_{U}}=(U, \mathcal{R} \cap U \times U, \mathcal{S} \cap U \times U)$ is a projection, and $\sigma\left(B A F_{\downarrow_{U}}\right)$ are the extensions of the projection.

P6. Global support If $a \in E$, then there must be an argument $b$ such that $b \in E$, and $b$ supports $a$.

P7. Grounded support If $a \in E$, then there must be an argument $b \in E$ and $(b, b) \in \mathcal{S}$ $(\operatorname{or}(a, a) \in \mathcal{S})$, such that there is a support sequence $\left(b, a_{0}, \ldots, a_{n}, a\right)$, all $a_{i} \in E$.

When analyzing an example with support relations, we can consider whether for this example the principles hold or not. The following table summarizes the relations between the reductions and the principles. See the technical report [20] for the proofs of these relations. 
Table 1. Comparison among the reductions and the proposed principles. We refer to Dung's semantics as follows: Complete $(\mathbb{C})$, Grounded $(\mathbb{G})$, Preferred $(\mathbb{P})$, Stable $(\mathbb{S})$. When a principle is never satisfied by a certain reduction for all semantics, we use the $\times$ symbol.

\begin{tabular}{|c|c|c|c|c|c|c|c|}
\hline Red & P1 & $\mathrm{P} 2$ & P3 & P4 & P5 & P6 & P7 \\
\hline RS & \multicolumn{2}{|c|}{$\mathbb{C} \mathbb{G P S} \mathbb{G} \mathbb{C P S}$} & $x$ & $\mathbb{G}$ & $\mathbb{C} \mathbb{G}$ & $\times$ & $\times$ \\
\hline RM & \multicolumn{2}{|c|}{$\mathbb{C} \mathbb{G P S} \mathbb{G} \mathbb{C P S}$} & $\times$ & $\mathbb{G}$ & $x$ & $x$ & $\times$ \\
\hline R2 & $\mathbb{C} \mathbb{G P S}$ & $\times$ & $\mathbb{G} \mathbb{C P S}$ & $\mathbb{G}$ & $\mathbb{C} \mathbb{G} \mathbb{P}$ & $\times$ & $\times$ \\
\hline RE & $\mathbb{C} \mathbb{G P S}$ & $\times$ & $\mathbb{G} \mathbb{C P S}$ & $\mathbb{G}$ & $x$ & $x$ & $\times$ \\
\hline $\mathrm{RD}$ & $\mathbb{C} \mathbb{G P S} \mathbb{C}$ & $\mathbb{C P S}$ & $\times$ & $\mathbb{G}$ & $x$ & $\times$ & $\times$ \\
\hline RN & $\mathbb{C} \mathbb{G P S}$ & $x$ & $\mathbb{G} \mathbb{C P S}$ & $\mathbb{G}$ & $x$ & $\times$ & $\times$ \\
\hline e- & $\mathbb{C} \mathbb{G P S}$ & $\times$ & $x$ & $\mathbb{G}$ & $x$ & $\mathbb{G} \mathbb{C P}$ & $\mathbb{C P}$ \\
\hline
\end{tabular}

\section{Divorce action}

Consider the bipolar framework visualized below (the detailed description of the divorce case and its scenarios comes after). The figure should be read as follows. A normal arrow visualizes attack, a dashed arrow visualizes support, a double box visualizes a prima facie argument which is self-supporting and single box visualizes a standard argument that does not support itself.



Figure 2. Divorce action

We first consider the graph with only the arguments in black and orange and the relations among them. The basic dilemma is represented by two arguments attacking each other, stating respectively that the child's best interest is that she lives with her mother $(M)$ or that the child's best interest is that she lives with her father $(F)$. Obviously, there may be additional reasons for these conclusions which we do not make explicit here, and in order to illustrate the dilemma-nature of the situation the judge has to deal with, we consider a well-balanced case. There are arguments attacking $(M)$ and $(F)$, and arguments attacking those attackers. If we do not consider support, then the grounded extension is $\{C J, W, O P, R\}$, two preferred extensions are $E_{1}=\{R, M, W, O P, C J\}$ and $E_{2}=\{F, C J, W, O P, R\}$. The judge cannot make any decision based on these two extensions, thus, further investigation is needed in this case.

In general, a support relation can be used to choose or select an extension, and this intuition is reflected by Principle P4 (short for P4 and the same for other principles). This 
so-called number of extensions principle says that adding support relations does not decrease the number of extensions. However, the analysis in Table 1 shows that this principle only holds trivially, for the grounded semantics. As there is only one extension under that semantics, the number of extensions can never increase. But for all other semantics, it is possible that the number of extensions grows when adding support relations. As we will see in the remainder of this section, that does not happen for this example.

The first support relation we interpret is the support of the father being wealthy $(W)$ to the argument that it is in the child's best interest that she lives with her father $(F)$. We leave the support of mother side in this following paragraph. There are different options for the interpretation of the support between $(W)$ and $(F)$. If the interpretation is deductive, then we add the supported attacks from $(W)$ to $(M)$ i.e., using RS, we have only one preferred extension $\{W, C J, O P, R, F\}$. If we add the mediated attack from $(M)$ to $(W)$ with the same interpretation but under RM, then we have two preferred extensions: $E_{1}=\{M, R, O P, C J\}$ and $E_{2}=\{R, F, W, C J, O P\}$. If we choose RD, we still have the two preferred extensions containing $(M)$ and $(F)$ separately. When we consider the interpretation of this support as necessary, saying, for example, that raising a child does take a lot of money, it means adding secondary or extended attack, since there is no attack coming from or going towards $(W)$, we have the same preferred extensions under these two reduction: $E_{1}=\{R, M, W, O P, C J\}$ and $E_{2}=\{F, C J, W, O P, R\}$. We see this as a clear case of deductive support, but as we saw, this doesn't solve the case either.

A similar analysis can be given using the P2 and P3 instead of the reductions, which as Table 1 shows are characteristic for deductive and necessary support respectively. P2 represents Closure and is characteristic for deductive support. It says that if $(W)$ is accepted then $(F)$ is accepted. So by contraposition, it means that if $(F)$ is not accepted, then also $(W)$ is not accepted. This implies that for the extension containing $(M)$, where $(F)$ is not accepted, by accepting closure, $(W)$ cannot be in the extension. Based on the above, the extensions show the preferred semantics under deductive reduction satisfies P2. However, in this scenario, the extensions still do not give decisive influence to the decision of the case. It may seem counterintuitive that under the deductive interpretation, a mediated attack is added from $(M)$ to $(W)$, as there does not seem to be a reason to question the wealth of the father. This surprising indirect attack is partly explained by P5, which shows that mediated attack does not satisfy BAF directionality. This reflects that the direction of the indirect attack goes against the direction of the attacks and supports in the framework.

If we consider the attacks and support of the $\operatorname{argument}(M)$, first we need to note that, according to the judicial practice and the public opinion, for decades, $(M)$ was taken for granted: judges automatically gave the custody for the mother, that is, $(M)$ was a prima facie argument and $(M)$ was the only argument being accepted. Thus, traditionally, $(M)$ could have been modeled as a self-supporting argument. However, the judicial practice and the public opinion have been changing, so in the figure above, we modeled $(M)$ as a standard argument requiring evidentiary support.

While the argument structure on the mother's side seems to be the same as the father's side, there is a difference coming from the law. The supporting argument might have a special status because of the rules of the Civil Code: the judge has to take the child's opinion into consideration when deciding about custody. The variants of the support interpretations and their relation to the interpretation of law can be shown with the analysis of this rule. We assume that the child wants to live with her mother $(O P)$. What 
does this mean? One can say that the obligation of taking an argument into consideration means that the $(O P)$ is prima facie and has to be accepted. If it is a prima facie argument, $(M)$ receives the evidentiary support it needs. But this in itself doesn't decide how argument $(O P)$ affects the extension. The extension depends on how we interpret the support relation between $(O P)$ and $(M)$ : deductive or necessary. It seems to be very intuitive to interpret the support relation deductive: the obligation of taking the opinion into consideration is apparently very much in align with what deductive support means: if we accept the opinion (which is prima facie) then we have to accept (M) too. But if we interpret the relation between $(O P)$ and $(M)$ deductive, under RS we have the only preferred extension $\{O P, C J, R, W\}$. Under RM we have the two preferred extensions $\{F, W, C J, R\}$ and $\{M, R, C J, O P\}$. These results in this scenario, on one hand, reflect the RS satisfies P8, because supported attack is directional. On the other hand, this result means that even the deductive support between the prima facie (OP) and (M), the fact that the child wants to live with her mother won't decide the case in the favor of her if there is some support on the father's side too. However, especially in such a well-balanced case, the judge's obligation to take the child's opinion into consideration might mean that it should be decisive. In order to show what that legal interpretation would mean formally, we need another approach. There is also a way to add the supported attack from $(O P)$ to $(F)$, and mediated attack from $(M)$ to $(W)$, by doing so we have the only preferred extension $\{R, O P, M, C J\}$. That is, in order to give the opinion a decisive nature, considering both relations between $(\mathrm{OP})$ and $(\mathrm{M})$ and $(\mathrm{W})$ and $(\mathrm{F})$ still deductive support, we do so under different reduction: using RS for (OP) and (M), and RM for the other. This context-dependent solution is needed to represent the given legal interpretation.

We now consider a scenario visualized in red and black in which $(O P)$ is attacked by (4): the child is only 4 years old and 4-year-olds don't know what they want. Argument (4) impairs that the child can form a reliable opinion at all, that is, (4) attacks $(O P)$. If the support between $(O P)$ and $(M)$ is deductive, under RS the unique preferred extension is $\{R, F, 4, C J, W\}$, while changes to $\{R, F, 4, C J, W\}$ and $\{R, 4, C J, M, O P\}$ under RM. If the interpretation of the support is necessary, under $\mathrm{R} 2$ the only extension should be $\{R, 4, F, C J, W\}$, while under RE, the extension is the same as the framework without considering support. The P3 Inverse Closure says that if $(M)$ is accepted then $(O P)$ is accepted. So by contraposition, it means that if $(O P)$ is not accepted, then also $(M)$ is not accepted. This implies that for the extension containing $(F)$, where $(M)$ is not accepted, by accepting Inverse Closure, $(O P)$ cannot be in the extension. Based on the above, the extensions show the preferred semantics under necessary interpretation satisfies P3.

Let's consider another scenario, as visualized in blue and black. In a Hungarian case, the court emphasized that the child's opinion is decisive concerning the custody, unless the child's healthy development would be endangered in the environment she would choose. This can be translated as the deductive nature of the support depends on whether there is a specific argument (of being endangered) attacking $(M)$. The child wants to live with her mother, but the mother often changes her boyfriends, and according to the judge, this would endanger the child's healthy emotional development $(D)$. If the interpretation of support is deductive and under RS, the only preferred extension is $\{R, 17, O P, C J, W\}$, the results under RM and RD are not decisive, either.

Finally, we consider a scenario visualized in green and black. The mother is a teacher, which supports that she knows how to handle children, and this again clearly supports that the child's best interest is to live with her mom. However, we also have the 
argument that mother often punishes the child harshly attacking $(K H)$. While the first support relation between $(T)$ and $(K H)$ is deductive, it seems reasonable to say the one between $(K H)$ and $(M)$ is necessary: it is difficult to defend a view as it is fine to give custody to someone who cannot handle children. $(M)$ receives secondary attack from $(P H)$ with the interpretation of necessary, if we still consider $(O P)$ supported attacks $(F)$, and the same for $(W)$ to $(M)$, both $(M)$ and $(F)$ should not be accepted. If we consider $(O P)$ mediated attacks $(F)$, and the same for $(W)$ to $(M),(F)$ is accepted in the only preferred semantics.

\section{Concluding remarks}

Polberg and Oren [14] aim to unify some of the most popular approaches to the representation of support and to construct an unified environment capable of handling the available types of support, and we agree with them that doing so not only provides important theoretical contributions, but also helps in the representation of real world domains. Towards this goal, we contribute to theory with a principle-based analysis for bipolar argumentation framework semantics, and to practice with a case study in divorce action. Our case study reveals a number of issues to be addressed in future work.

First of all, once the support relations are interpreted, it seems that we need to consider bipolar argumentation with multiple support relations $B A F=\left\langle\mathcal{A}, \mathcal{R}, \mathcal{S}_{1}, \ldots, \mathcal{S}_{n}\right\rangle$ and adapt the definitions accordingly. Moreover, for the interpretation of the support relations, we can define new combinations. For example, it is quite natural for a support to be both deductive and evidentiary, or to be both necessary and evidentiary. This can be achieved by combining definitions 2 and 3 in the following way. We first add indirect attacks using the construction of definition 2 , and then we consider evidentiary semantics instead of Dung semantics. In other words, the support relation is used twice, first to define indirect attacks, and then again to define e-admissible sets, and so on. This would lead to six more rows in Table 1, for each of the reductions now combined with evidentiary support, leading to a total of 13 rows. Such combined interpretations of support can be characterized by new principles combining on the one hand closure and inverse closure, characteristic for deductive and necessary support, and on the other hand grounded or global support. For example:

P8. Self-supported Closure For all $B A F=(\mathcal{A}, \mathcal{R}, \mathcal{S})$, for all extensions $\mathrm{E}$ in $\mathcal{E}_{\mathcal{S} \mathcal{R}}$, $\forall a, b \in \mathcal{A}$, if $a S a, a S b$ and $a \in E$, then $b \in E$.

P9. Self-supported Inverse Closure For all $B A F=\langle\mathcal{A}, \mathcal{R}, \mathcal{S}\rangle$, for all extensions $\mathrm{E}$ in $\mathcal{E}_{\mathcal{S} \mathcal{R}}, \forall a, b \in \mathcal{A}$, if $a \mathcal{S} a, a S b$ and $b \in E$, then $a \in E$.

More generally, sometimes it seems to depend on the context whether a support is interpreted as deductive or necessary, like the interpretation of the support between $(O P)$ and $(M)$ in the running example. Moreover, in our experience in modeling the divorce action, the choice between deductive, necessary and evidentiary support is rather limited. This reinforces the results on the theory-based validation of existing kinds of support, where only for necessary support some results have been obtained $[17,5,18]$. Alternative approaches are proposed by Gabbay [7], Gottifredi et al [8] and Potyka [15]. The principle-based analysis suggests that there are many possible combinations which have not been explored yet within the realm of bipolar argumentation. We also found that 
in this example, the necessary supports didn't affect much the outcome: compared to the original framework, the result stayed the same. However, in such a symmetric, what is more, parallel case, where the two arguments constituting the dilemma by attacking each other are the same with different subjects, considering a support necessary on one side should result in an attack on the other: if knowing how to handle children is necessary on the mother side, it is on the father side too, which if he lacks, it should be added as an attack. This indirect attack nature of the necessary support is of course contextdependent, but its formal representation might be worth further investigation.

Second, we found that in modeling the divorce action, there are two ways to model support. On the one hand we can model both attacks and supports among arguments, as we did in this paper, but on the other hand we can also model all support within the arguments, and only attack among the arguments. It seems that in the former case, most authors see the need to generalize to sets of arguments attacking or supporting arguments, as in dialectical argumentation frameworks. Despite this apparently fundamental modeling choice, we found little help in the literature about the advantages and disadvantages of the two approaches. Also it is unclear to us in general whether we can translate one kind of model into the other and vice versa. Consider the scenario where the child is only 4 years old. (4) actually rather attacks the deductive support between $(O P)$ and $(M)$. Now consider that the attorney of the mother would like bring some arguments regarding the wealth difference between the parents. He might say: "money is not everything". What would this argument attack? It wouldn't attack the fact that the father is wealthy. What it would attack is the the deductive support relation between $(W)$ and $(F)$. Consider a scenario where the father is wealthy because he is an entrepreneur. This as an argument, or if we consider it as two arguments one deductively supporting the other, the support relation between them could be attacked by the mother's attorney's argument as "entrepreneur is risky". In this paper we didn't use a language for describing arguments attacking relations, but we think it might be fruitful research direction investigating comparing solutions.

Third, when defining our principles we found a lot of research on semantics and principles for complex forms with set attack and set support, and numbers representing the strengths and supports [1]. From a formal perspective, we believe that this research is very useful because it helps relate formal argumentation to other domains like multicriteria decision making.

Fourth, what struck us in this research is the similarity between reductions for bipolar argumentation frameworks, and the for preference-based argumentation frameworks we studied in earlier work. Whereas in both frameworks, the support relation and the preference can be both added and removed. Moreover, we found that the theory of reductions for preference based argumentation and bipolar argumentation is closely related to dynamic principles for AF [19], and we expect that this can be a source of further principles. Finally, like in preference-based argumentation, we believe that bipolar frameworks with symmetric attacks can be studied as a fragment with good computational properties.

\section{References}

[1] Leila Amgoud and Jonathan Ben-Naim. Evaluation of arguments in weighted bipolar graphs. Int. J. Approx. Reason., 99:39-55, 2018. 
[2] Guido Boella, Dov M. Gabbay, Leendert van der Torre, and Serena Villata. Support in abstract argumentation. In Pietro Baroni, Federico Cerutti, Massimiliano Giacomin, and Guillermo Ricardo Simari, editors, Computational Models of Argument: Proceedings of COMMA 2010, Desenzano del Garda, Italy, September 8-10, 2010, volume 216 of Frontiers in Artificial Intelligence and Applications, pages 111-122. IOS Press, 2010.

[3] Claudette Cayrol and Marie-Christine Lagasquie-Schiex. Bipolarity in argumentation graphs: Towards a better understanding. International Journal of Approximate Reasoning, 54(7):876-899, 2013.

[4] Claudette Cayrol and Marie-Christine Lagasquie-Schiex. An axiomatic approach to support in argumentation. In International Workshop on Theory and Applications of Formal Argumentation, pages 74-91. Springer, 2015.

[5] Andrea Cohen, Simon Parsons, Elizabeth I. Sklar, and Peter McBurney. A characterization of types of support between structured arguments and their relationship with support in abstract argumentation. Int. J. Approx. Reason., 94:76-104, 2018.

[6] Phan M. Dung. On the acceptability of arguments and its fundamental role in non-monotonic reasoning, logic programming and n-person games. Artificial Intelligence, 77:321-357, 1995.

[7] Dov M. Gabbay. Logical foundations for bipolar and tripolar argumentation networks: preliminary results. J. Log. Comput., 26(1):247-292, 2016.

[8] Sebastian Gottifredi, Andrea Cohen, Alejandro Javier García, and Guillermo Ricardo Simari. Characterizing acceptability semantics of argumentation frameworks with recursive attack and support relations. Artif. Intell., 262:336-368, 2018.

[9] Farid Nouioua and Vincent Risch. Bipolar argumentation frameworks with specialized supports. In 2010 22nd IEEE International Conference on Tools with Artificial Intelligence, volume 1, pages 215-218. IEEE, 2010.

[10] Nir Oren, Michael Luck, and Chris Reed. Moving between argumentation frameworks. In Proceedings of the 2010 International Conference on Computational Models of Argument. IOS Press, 2010.

[11] Nir Oren and Timothy J. Norman. Semantics for evidence-based argumentation. In Philippe Besnard, Sylvie Doutre, and Anthony Hunter, editors, Computational Models of Argument: Proceedings of COMMA 2008, Toulouse, France, May 28-30, 2008, volume 172 of Frontiers in Artificial Intelligence and Applications, pages 276-284. IOS Press, 2008.

[12] Sylwia Polberg. Intertranslatability of abstract argumentation frameworks. Technical report, Cardiff University, 2017.

[13] Sylwia Polberg and Anthony Hunter. Empirical evaluation of abstract argumentation: Supporting the need for bipolar and probabilistic approaches. Int. J. Approx. Reason., 93:487-543, 2018.

[14] Sylwia Polberg and Nir Oren. Revisiting support in abstract argumentation systems. In Simon Parsons, Nir Oren, Chris Reed, and Federico Cerutti, editors, Computational Models of Argument - Proceedings of COMMA 2014, Atholl Palace Hotel, Scottish Highlands, UK, September 9-12, 2014, volume 266 of Frontiers in Artificial Intelligence and Applications, pages 369-376. IOS Press, 2014.

[15] Nico Potyka. Bipolar abstract argumentation with dual attacks and supports.

[16] Henry Prakken. Logical tools for modelling legal argument: a study of defeasible reasoning in law, volume 32. Springer Science \& Business Media, 2013.

[17] Henry Prakken. On support relations in abstract argumentation as abstractions of inferential relations. In Torsten Schaub, Gerhard Friedrich, and Barry O'Sullivan, editors, ECAI 2014 - 21st European Conference on Artificial Intelligence, 18-22 August 2014, Prague, Czech Republic - Including Prestigious Applications of Intelligent Systems (PAIS 2014), volume 263 of Frontiers in Artificial Intelligence and Applications, pages 735-740. IOS Press, 2014.

[18] Henry Prakken. On validating theories of abstract argumentation frameworks: the case of bipolar argumentation frameworks. In Proceedings of the 8th Workshop on Computational Models of Natural Argument (CMNA 2020), Perugia, Italy (and online). CEUR-WS.org, 2020.

[19] Tjitze Rienstra, Chiaki Sakama, and Leendert van der Torre. Persistence and monotony properties of argumentation semantics. In International Workshop on Theory and Applications of Formal Argumentation, pages 211-225. Springer, 2015.

[20] Liuwen Yu and Leendert Van der Torre. A principle-based approach to bipolar argumentation. Available at https://nmr2020.dc.uba.ar/WorkshopNotes.pdf (2020/09/20). 